\title{
Dephasing due to background charge fluctuations
}

\author{
Toshifumi Itakura and Yasuhiro Tokura \\ NTT Basic Research Laboratories, NTT Corporation \\ 3-1, Morinosato Wakamiya, Atsugi-shi, Kanagawa Pref., 243-0198 Japan \\ Tel: +81-46-240-3938 Fax: +81-46-240-4726 \\ E-mail: itakura@will.brl.ntt.co.jp
}

(recieved March 18, 2002. accepted on March 20, 2003)

\begin{abstract}
In quantum computation, quantum coherence must be maintained during gate operation. However, in physical implementations, various couplings with the environment are unavoidable and can lead to a dephasing of a quantum bit (qubit). The background charge fluctuations are an important dephasing process, especially in a charge qubit system. We examined the dephasing rate of a qubit due to random telegraph noise. Solving stochastic differential equations, we obtained the dephasing rate of a qubit constructed of a coupled-dot system; we applied our results to the charge Josephson qubit system. We examined the dephasing rates due to two types of couplings between the coupled-dot system and the background charge, namely, fluctuation in the tunnel coupling constant and fluctuation in the asymmetric bias. For a strong coupling condition, the dephasing rate was inversely proportional to the time constant of the telegraph noise. When there is fluctuation in the tunnel coupling constant, Gaussian decay occurs in the initial regime. We also examined the rate of dephasing due to many impurity sites. For a weak coupling condition with fluctuation in the asymmetric bias, the obtained dephasing rate coincided with that obtained by the perturbation method using the spectral weight of a boson thermal bath, which is proportional to the inverse of the frequency.
\end{abstract}

PACS numbers: 03.65.Yz, 73.21.La, 73.23-b

\section{INTRODUCTION}

Efforts to implement quantum computation have recently intensified. The application of the quantum bit (qubit) to solid-state materials, such as superconducting Josephson junctions [1] and quantum dots [2-4], is particularly promising, because these implementations have the advantage of scalability. In a coupled-dot system, for example, the localized states in the left and right dots are treated as a basic two-level system, in which the tunnel coupling between the two dots constructs a quantum superposition of the dots. This superposition manifests itself in coherent quantum oscillation (Rabi oscillation), and transition can be induced between the superposed states [5]. A nanometer-scale superconducting electrode connected to a reservoir via a Josephson junction constitutes an artificial two-level system in which the charge states, coupled by tunneling, differ by $2 e$, where $e$ is the electronic charge. This system has shown clear Rabi oscillation [1].

Quantum coherence must be maintained during quantum gate operation. Dephasing, characterized by the dephasing time, originates from various couplings between the qubit and the environment. When the qubit is implemented in a solid-state system, the effects of phonons and electromagnetic and background charge fluctuations (BCFs) are important in the dephsing process. The effect of phonons has been examined in semiconductor quantum dots as the source of the dephasing accompanying dissipation [6]; the effect of electromagnetic fluctuation in
Josephson junction qubits has been extensively studied [7]. However, BCFs have not yet been examined systematically, in spite of their importance in the dephasing process.

BCFs have been observed in many systems [8-11]. In nanoscale systems, they are the electrostatic potential fluctuations due to the dynamics of electrons or holes trapped at impurity sites. In particular, the charge of a single impurity fluctuates with the Lorentzian spectrum form, which is called "random telegraph noise" in the time domain $[10,12]$. The random distribution of the positions of such impurities and their time constants lead to BCFs or $1 / f$ noise [13]. In solid-state charge qubits, these BCFs lead to a dynamical electrostatic disturbance and hence the dephasing. The effect of $1 / \mathrm{f}$ noise on a charge Josephson qubit has been examined theoretically - the interaction between the qubit and environment has been treated by the perturbation method $[14,15]$, by Gaussian approximation [16] and by the path integral method within a spin-Boson model $[14,15]$. When fluctuating impurities exist in the substrate, not in the junction [11], the coupling between the qubit and BCFs is weak, and the perturbation method is sufficient. However, when the interaction between the qubit and environment is strong, methods that go beyond perturbation are needed.

In this study, we investigated how the electrostatic disturbance of time constant $\tau_{0}$ coming from a single impurity affects the quantum coherence of a qubit irrespective of the strength of the qubit-impurity coupling. We also examine the effect of many impurity sites. This approach 
is in clear contrast with previous ones $[16,15]$, in which the phenomenological spectral weight of the boson thermal bath was used to characterize the effect of BCFs.

We consider two types of couplings between the qubit and the environment: pure dephasing and dephasing accompanied with relaxation of the population [17]. In symmetrical coupled-dot systems, the former corresponds to a fluctuation in the tunnel coupling constant, and the latter to that in the asymmetric bias $[18,19]$. The mapping from a coupled-dot system to the Josephson charge qubit is discussed in Sec. VI. By using the method of stochastic differential equations, we obtain analytically the dephasing rate, which is shown to be always smaller than $\tau_{0}^{-1}$. It should be noted that this dephasing process does not mean the qubit becomes entangled with the environment, but rather it means the stochastical evolution of an external classical field, suppressing the off-diagonal density matrix elements of the qubit after being averaged over statistically distributed samples.

Section II defines the Hamiltonian of the system. Section III explains the method of stochastic differential equations. The fluctuations in tunnel coupling and asymmetric bias are examined in Secs. IV and V, respectively. Section VI is devoted to discussion, including the effect of many impurities. Section VII summarizes the paper.

\section{HAMILTONIAN}

The qubit and the effect of a single impurity are examined in terms of the following Hamiltonian:

$$
\begin{aligned}
\mathcal{H} & =H_{q b}+H_{q b-i m p}, \\
H_{q b} & =\frac{\hbar \Delta}{2}\left(c_{L}^{\dagger} c_{R}+c_{R}^{\dagger} c_{L}\right)+\frac{\hbar \epsilon}{2}\left(c_{L}^{\dagger} c_{L}-c_{R}^{\dagger} c_{R}\right),
\end{aligned}
$$

where $c_{L, R}^{\dagger}$ and $c_{L, R}$ are the creation and annihilation operators of the left and the right dots, assuming a single level for each dot, as shown in Fig. 1(a). The $\Delta$ is the tunnel coupling between the dots, and $\epsilon$ is the asymmetric bias between them. The interaction between a qubit and the charge at the impurity site is described by the following Hamiltonian:

$$
\begin{aligned}
H_{q b-i m p} & =\frac{\hbar J_{T}}{2}\left(c_{L}^{\dagger} c_{R}+c_{R}^{\dagger} c_{L}\right) 2\left(d^{\dagger} d-1 / 2\right) \\
& +\frac{\hbar J_{B}}{2}\left(c_{L}^{\dagger} c_{L}-c_{R}^{\dagger} c_{R}\right) 2\left(d^{\dagger} d-1 / 2\right)
\end{aligned}
$$

where $J_{T}$ is the magnitude of the fluctuation in the tunnel coupling, $J_{B}$ is the magnitude of the fluctuation in the asymmetrical bias, and $d^{\dagger}$ and $d$ are the creation and annihilation operators of the charge at the impurity site, respectively.

\section{qubit}
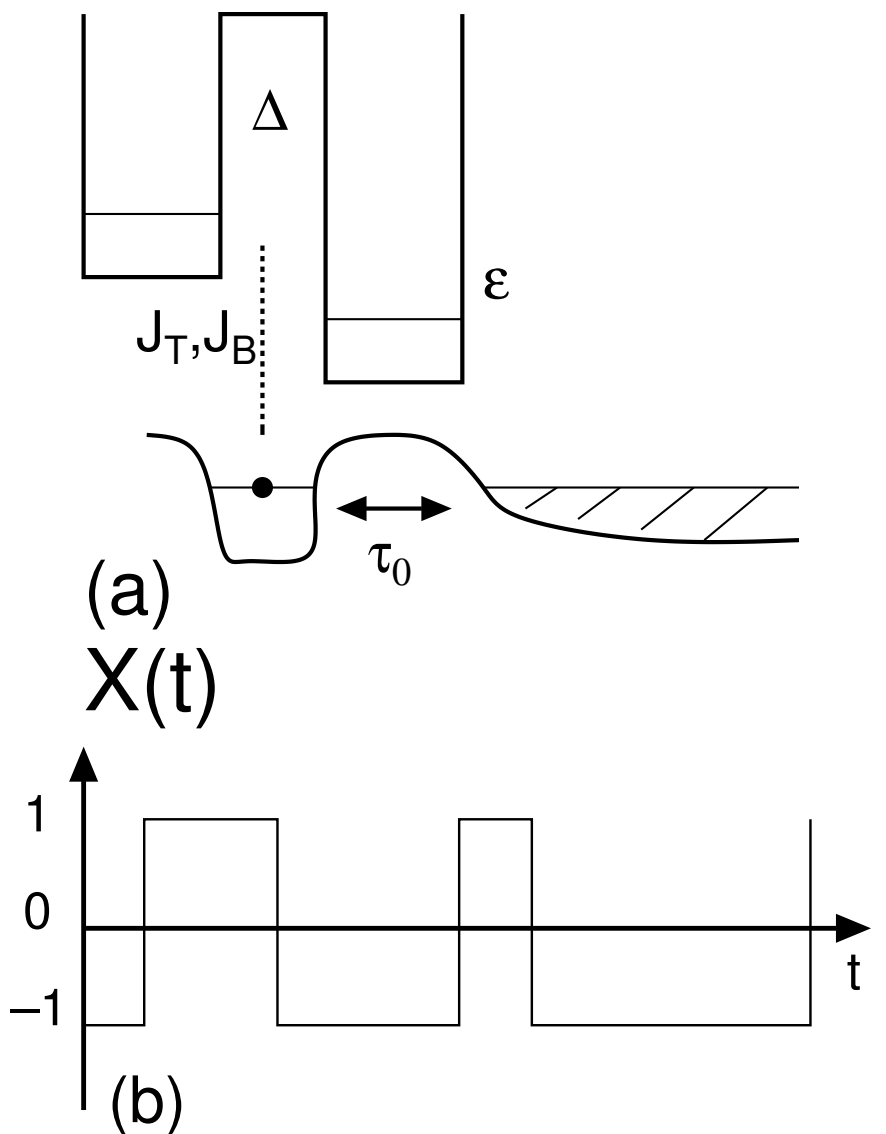

FIG. 1. (a) Schematic diagram of coupled dots and environment constituting an impurity site and the electron reservoir. (b) Example time sequence of random telegraph noise.

We assume that the time evolution of statistical variable $X(t)\left(=2<d^{\dagger} d-1 / 2>\right)$ is a Poisson process. Assuming a strong coupling between the charge at the impurity site and the nearby electron reservoir, the dynamics of the charge induces not Gaussian white noise, but random telegraph noise $[12,18,19]$. We therefore consider the effect of random telegraph noise with characteristic time constant $\tau_{0}$, where the statistical variable $X(t)$ takes the value 1 or -1 (Fig. 1(b)).

The time constant is determined by the barrier height of the electron or hole trap and the temperature, like $\tau_{0}=$ $A e^{W / k_{B} T}$, where $W, k_{B}, T$, and $A$ are the activation energy of the impurity potential, Boltzmann's constant, the temperature, and the temperature-independent prefactor, respectively [20]. When the temperature decreases, $\tau_{0}$ becomes longer. The telegraph noise has been experimentally observed to take a value of 1 or -1 with asymmetric probabilities, which arises from the difference between the Fermi energy of the electron reservoir and the energy level of the impurity sites. [12]. To include this asymmetric weight, we introduce asymmetric probabil- 
ities $p_{u}$ and $p_{d}$, which relate the asymmetric transition rates for the process from -1 to $1\left(\tau_{u}^{-1}=\left(p_{u} \tau_{0}\right)^{-1}\right)$ to those of the opposite process $\left(\tau_{d}^{-1}=\left(p_{d} \tau_{0}\right)^{-1}\right)$.

We neglect the backaction from the qubit to the charge at the impurity site, so this environment reduces to a classical stochastic external field. We also assume that the temperature is high enough for the effect of the quantum fluctuation of the charge between the impurity site and electron reservoir to be neglected.

For compactness, we rewrite the Hamiltonian in terms of the Pauli matrices while rotating basis $\pi / 2$ from the basis of localized states in the left and right dots to bonding-antibonding basis:

$$
\mathcal{H}=\frac{\hbar \Delta}{2} \sigma_{z}+\frac{\hbar \epsilon}{2} \sigma_{x}+\frac{\hbar J_{T}}{2} \sigma_{z} X(t)+\frac{\hbar J_{B}}{2} \sigma_{x} X(t) .
$$

In the following, we consider only the case of a symmetrical coupled-dot system, $\epsilon=0$, in which the effect of the bias fluctuation due to the dephasing starts only from the second order, $J_{B}^{2}$, as shown in the following, and is less effective in the perturbation regime. As reduced Hamiltonian Eq. (4) suggests, the present results can also be applied to other quantum two-level systems in which telegraph-type fluctuation exists (see Sec. VI).

\section{METHOD}

We are interested in the time-evolution of the qubit's two-by-two density matrix, $\rho(t)$, with an arbitrary initial condition at $t=0, \rho(0)$. If $\mathrm{BCF}$ is absent, starting with the initial condition that the left dot is occupied, for example, the density matrix at $t=0$ is given by

$$
\rho(t=0)=W\left(\frac{\pi}{2}\right)\left(\begin{array}{ll}
1 & 0 \\
0 & 0
\end{array}\right) W^{\dagger}\left(\frac{\pi}{2}\right)
$$

where $W\left(\frac{\pi}{2}\right)$ is the matrix of rotation from the localized basis to the bonding-antibonding basis. At time t,

$$
\begin{aligned}
\rho(t) & =e^{-i \frac{t}{2} \Delta \sigma_{z}} W\left(\frac{\pi}{2}\right)\left(\begin{array}{ll}
1 & 0 \\
0 & 0
\end{array}\right) W^{\dagger}\left(\frac{\pi}{2}\right) e^{i \frac{t}{2} \Delta \sigma_{z}} \\
& =W\left(\frac{\pi}{2}\right)\left(\begin{array}{cc}
\frac{1+\cos \Delta t}{2} & i \sin \Delta t \\
-i \sin \Delta t & \frac{1-\cos \Delta t}{2}
\end{array}\right) W^{\dagger}\left(\frac{\pi}{2}\right),
\end{aligned}
$$

where the bases of the inner matrix are the left and right occupancy states. Therefore, the density matrix shows Rabi oscillation with frequency $\Delta$.

In the following, we keep the matrix indices in the bonding-antibonding basis. To examine the instantaneous potential change, we use the method of stochastic differential equations [21]. The density matrix averaged over all possible sequences of telegraph noise can be represented as a series:

$$
\begin{aligned}
\rho(t) e^{t / \tau_{0}}= & \sum_{k=0}^{\infty} \frac{1}{\tau_{0}^{k}} \int_{0}^{t} d t_{k} \int_{0}^{t_{k}} d t_{k-1} \ldots \int_{0}^{t_{2}} d t_{1} \\
\times & \int_{X} d W\left(X_{k}\right) \int_{X} d W\left(X_{k-1}\right) \ldots \\
& \int_{X} d W\left(X_{0}\right) \rho\left(t, t_{k}\right),
\end{aligned}
$$

where $d W(X)$ is the distribution of $X$ with the probability of $p_{u}$ for $X=1$ and that of $p_{d}$ for $X=-1$, with the constraint $p_{u}+p_{d}=1$. The density matrix before the ensemble average, $\rho\left(t, t_{k}\right)$, is given by

$$
\begin{aligned}
& \rho\left(t, t_{k}\right)=S\left(X_{k} ; t, t_{k}\right) \cdots S\left(X_{1} ; t_{2}, t_{1}\right) S\left(X_{0} ; t_{1}, 0\right) \\
& \quad \times \rho(0) S^{-1}\left(X_{0} ; t_{1}, 0\right) S^{-1}\left(X_{1} ; t_{2}, t_{1}\right) \cdots S^{-1}\left(X_{k} ; t, t_{k}\right),
\end{aligned}
$$

where $S\left(X ; t, t^{\prime}\right)=S\left(X, t-t^{\prime}\right)$ is the unitary time evolution operator, which is determined by

$$
i \hbar \frac{d S(X, t)}{d t}=\mathcal{H}(X) S(X, t)
$$

The explicit form of $S(X, t)$ is given by

$$
\begin{aligned}
& S(X, t) \\
= & \left(\begin{array}{cc}
\cos \frac{1}{2} a t-i\left(\frac{\Delta+J_{T} X}{a}\right) \sin \frac{1}{2} a t & -i \frac{J_{B} X}{a} \sin \frac{1}{2} a t \\
-i \frac{J_{B} X}{a} \sin \frac{1}{2} a t & \cos \frac{1}{2} a t+i \frac{\Delta+J_{T} X}{a} \sin \frac{1}{2} a t
\end{array}\right),
\end{aligned}
$$

where $a=\sqrt{\left(\Delta+J_{T} X\right)^{2}+J_{B}^{2}}$. Equation (8) can be rewritten in terms of the integral equation

$$
\begin{aligned}
\rho(t) e^{\tau / \tau_{0}} & =\int_{X} S(X ; \tau, 0) \rho(0) S^{-1}(X ; \tau, 0) d W(X) \\
& +\frac{1}{\tau_{0}} \int^{\tau} e^{t / \tau_{0}} \int_{X} S(X ; \tau, t) \rho(t) S^{-1}(X ; \tau, t) d W(X) d t .
\end{aligned}
$$

Using $S(X ; \tau, t)$, we define matrix $R^{i m}(\tau, t)$ as follows:

$$
\begin{aligned}
& R_{l k}^{i m}(\tau, t)=\int_{X} S_{i k}(X ; \tau, t) S_{l m}^{-1}(X ; \tau, t) d W(X), \\
& R_{l k}^{i m}(\tau, t)=\left(R_{k l}^{m i}\right)^{*}(\tau, t) .
\end{aligned}
$$

We can then reduce Eq. (12) to the following compact form:

$$
\begin{aligned}
& \rho_{i m}(\tau)=e^{-\tau / \tau_{0}} \operatorname{Tr}\left[R^{i m}(\tau, 0) \rho(0)\right] \\
& \quad+\frac{1}{\tau_{0}} \int_{0}^{\tau} d t \exp \left[-\frac{\tau-t}{\tau_{0}}\right] \operatorname{Tr}\left[R^{i m}(\tau, t) \rho(t)\right] .
\end{aligned}
$$




\section{FLUCTUATION IN TUNNEL COUPLING}

First, we consider the case of fluctuation in tunnel coupling $\left(J_{T} \neq 0, J_{B}=0\right)$. Since the interaction Hamiltonian commutes with $H_{q b}$, the environment leads to pure dephasing without energy dissipation. We derive $S(X, t)$ and $R_{l k}^{i m}(t)$ from Eqs. (11) and (13) as follows:

$$
\begin{aligned}
S_{k l}(X, \tau-t) & =\exp \left[\frac{i}{2}\left(\Delta(\tau-t)-J_{T} X(\tau-t)\right)(-1)^{k}\right] \delta_{k l} \\
R_{l k}^{n m}(\tau-t) & =\int_{X} \exp \left[i \Delta(\tau-t)+i J_{T} X(\tau-t)\right] \\
& \times \delta_{n k} \delta_{l m} d W(X) .
\end{aligned}
$$

As a result, the off-diagonal element of the density matrix obeys the following integral equation:

$$
\sigma(\tau)=e^{-\tau / \tau_{0}} R(\tau)+\frac{1}{\tau_{0}} \int_{0}^{\tau} R(\tau-t) \exp \left[-\frac{\tau-t}{\tau_{0}}\right] \sigma(t) d t,
$$

where $\sigma(\tau)=e^{-i \Delta \tau} \rho_{12}(\tau) /\left|\rho_{12}(0)\right|$ is a normalized offdiagonal element of the qubit density matrix measured in a Rabi oscillation frame, and

$$
R(\tau-t)=p_{u} \exp \left[i J_{T}(\tau-t)\right]+p_{d} \exp \left[-i J_{T}(\tau-t)\right] .
$$

Equation (18) can be rewritten as a differential equation:

$$
\sigma^{\prime \prime}(\tau)+\frac{1}{\tau_{0}} \sigma^{\prime}(\tau)+\left(J_{T}^{2}+i\left(\frac{p_{u}-p_{d}}{\tau_{0}}\right) J_{T}\right) \sigma(\tau)=0 .
$$

The initial conditions are $\sigma(0)=e^{i \phi}=\rho_{12}(0) /\left|\rho_{12}(0)\right|$ and $\sigma^{\prime}(0)=0$. As a result of coupling with the environment, the off-diagonal element of the density matrix decays as a function of time. When the real part of the two roots of the characteristic equation of Eq. (20) almost completely degenerates, the short-time behavior for $t<\min \left(\frac{\sqrt{2}}{J_{T}}, 3 \tau_{0}\right)$ (initial regime) is not a simple exponential decay. In this initial regime, the offdiagonal element of the density matrix shows Gaussian decay, $\sigma(t) \sim \sigma(0)\left(1-J_{T}^{2} t^{2} / 2+\cdots\right) \simeq \sigma(0) e^{-J_{T}^{2} t^{2} / 2}$, irrespective of the asymmetric probabilities, $p_{u}$ and $p_{d}$. The decay of the off-diagonal element of the density matrix becomes exponential for the asymptotic regime, $t \gg\left(1 /\left(\sqrt{\mid \frac{1}{\tau_{0}^{2}}-4 J_{T}^{2}} \mid\right)\right.$, when $p_{u}=p_{d}$. For $J_{T} \tau_{0} \ll 1$, this criterion is obtained when one of the two exponential decay terms becomes negligibly small. For $J_{T} \tau_{0} \gg 1$, the time constants of the envelope of the two dumped oscillating terms are the same, $1 /\left(2 \tau_{0}\right)$. Exponential decay appears after the inverse of the oscillation frequency:
$1 / \sqrt{\left|4 J_{T}^{2}-\frac{1}{\tau_{0}^{2}}\right|}$. When $p_{u}=p_{d}$ and $J_{T} \tau_{0}=\frac{1}{2}$, one obtains, $\sigma(t)=e^{-\frac{t}{4 \tau_{0}}}\left(1+\frac{t}{2 \tau_{0}}\right)$, where the dephasing can never be a simple exponential decay.

The time constant of this exponential decay corresponds to the dephasing time, $T_{2}$. For $p_{u} \neq p_{d}$ [18],

$$
T_{2}^{-1}=\frac{1}{2} \Re\left[\frac{1}{\tau_{0}}-\sqrt{\left(\frac{1}{\tau_{0}}\right)^{2}-4 J_{T}^{2}-4 i\left(\frac{p_{u}-p_{d}}{\tau_{0}}\right) J_{T}}\right] .
$$

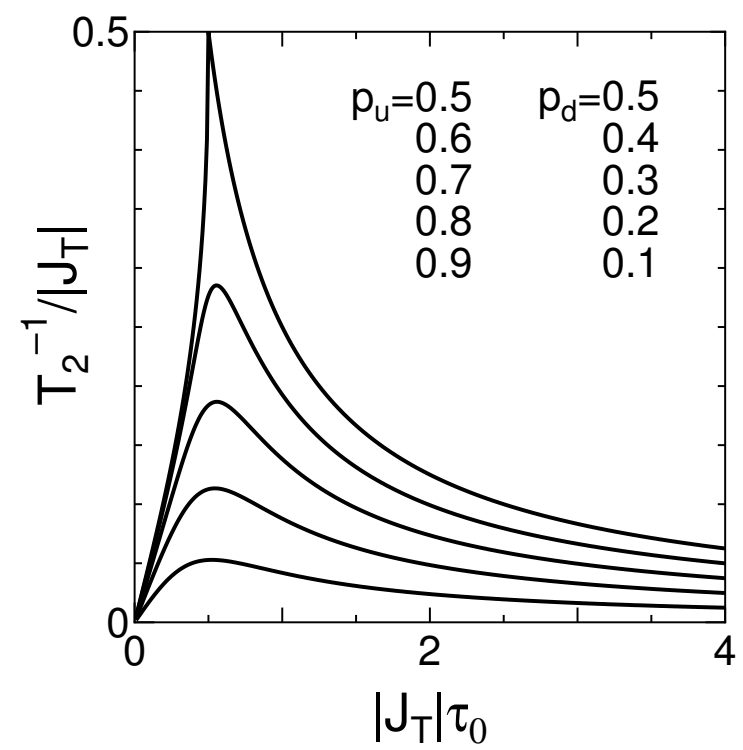

FIG. 2. Dependence of dephasing rate $T_{2}^{-1} /\left|J_{T}\right|$ on tunnel coupling constant $\left|J_{T}\right| \tau_{0}$ for various values of $p_{u}$ and $p_{d}$ with $J_{T} \neq 0$ and $J_{B}=0$.

Figure 2 shows the $\left|J_{T}\right| \tau_{0}$ dependence of dephasing rate $T_{2}^{-1}$ for $p_{u}=0.5,0.6,0.7,0.8$, and 0.9. In the two limits of weak and strong coupling, we have

$$
T_{2}^{-1}=\left\{\begin{array}{rr}
\left(1-\left(p_{u}-p_{d}\right)^{2}\right) J_{T}^{2} \tau_{0}, & 1 / \tau_{0} \gg\left|J_{T}\right| \\
\left(1-\left|p_{u}-p_{d}\right|\right) / 2 \tau_{0}, & 1 / \tau_{0} \ll\left|J_{T}\right|
\end{array}\right.
$$

Namely, for a fixed $\left|J_{T}\right|$, when $\tau_{0}$ increases from $0, T_{2}^{-1}$ first increases and then decreases. It has a single maximum of $\left(1-\sqrt{\left|p_{u}-p_{d}\right|}\right)\left|J_{T}\right|$ when $\tau_{0}=1 /\left(2\left|J_{T}\right|\right)$; therefore, for any parameters, $T_{2}>2 \tau_{0}$. Changing weight $p_{u}$ to make it more asymmetric reduces $T_{2}^{-1}$. In the limit of $p_{u} \rightarrow 0$ or 1 , the environment is nearly frozen, so the dephasing time becomes infinity.

\section{FLUCTUATION IN ASYMMETRIC BIAS}

Next we examine the effect of bias fluctuation $\left(J_{T}=\right.$ $\left.0, J_{B} \neq 0\right)$. We consider only the case of symmetrically weighted telegraph noise $\left(p_{u}=p_{d}=1 / 2\right)$ for simplicity. In this model, $H_{q b}$ and the interaction Hamiltonian do not commute and the dephasing process is accompanied by a relaxation of the population. The unitary operator is thus 


$$
\begin{aligned}
& S(X, t) \\
= & \left(\begin{array}{cc}
\cos \frac{1}{2} \Omega t-i \frac{\Delta}{\Omega} \sin \frac{1}{2} \Omega t & -i \frac{J_{B} X}{\Omega} \sin \frac{1}{2} \Omega t \\
-i \frac{J_{B} X}{\Omega} \sin \frac{1}{2} \Omega t & \cos \frac{1}{2} \Omega t+i \frac{\Delta}{\Omega} \sin \frac{1}{2} \Omega t
\end{array}\right),
\end{aligned}
$$

where $\Omega=\sqrt{\Delta^{2}+J_{B}^{2}}$ is the nutation frequency. After averaging, the few non-vanishing elements of matrix $R_{l k}^{i m}(t)$ have the form

$$
\begin{aligned}
R_{11}^{11}(\tau-t)= & R_{22}^{22}(\tau-t)=1-P_{12}(\tau-t), \\
R_{22}^{11}(\tau-t)= & R_{11}^{22}(\tau-t)=R_{12}^{12}(\tau-t)=R_{21}^{21}(\tau-t) \\
= & P_{12}(\tau-t), \\
R_{21}^{12}(\tau-t)= & R_{12}^{21 *}(\tau-t)=\left[P_{12}(\tau-t)+\cos \Omega(\tau-t)\right. \\
& \left.+\frac{\Delta}{\Omega} \sin \Omega(\tau-t)\right],
\end{aligned}
$$

where

$$
P_{12}(\tau-t)=\frac{J_{B}^{2}}{\Omega^{2}} \sin ^{2} \frac{\Omega(\tau-t)}{2} .
$$

The time evolution of the diagonal element of this matrix is determined by the following differential equation $[21,22]$ :

$n^{\prime \prime \prime}(\tau)+\frac{2}{\tau_{0}} n^{\prime \prime}(\tau)+\left(\frac{1}{\tau_{0}^{2}}+\Delta^{2}+J_{B}^{2}\right) n^{\prime}(\tau)+\frac{J_{B}^{2}}{\tau_{0}} n(\tau)=0$.

Here, $n(\tau)=\rho_{11}-\rho_{22}$, and the initial conditions are $n^{\prime \prime}(0)=-J_{B}^{2} n(0)$ and $n^{\prime}(0)=0$; and $n(0)$ depends on the initial population having an arbitrary value between 1 and -1 . The time constant of the exponential decay of $n(\tau)$ is the relaxation time of the population, $T_{1}$. When $\left|J_{B}\right| \ll \Delta, 1 / \tau_{0}$ (weak coupling), $T_{1}^{-1} \sim J_{B}^{2} \tau_{0}$. When $\left|J_{B}\right| \gg \Delta, 1 / \tau_{0}$ (strong coupling), $T_{1}^{-1} \sim 1 / 2 \tau_{0}$.

The obtained differential equation for the off-diagonal element is

$$
\begin{gathered}
\sigma_{12}^{\prime \prime \prime}(\tau)+\frac{2}{\tau_{0}} \sigma_{12}^{\prime \prime}(\tau)+\left(\frac{1}{\tau_{0}^{2}}+\Delta^{2}+J_{B}^{2}+i \frac{\Delta}{\tau_{0}}\right) \sigma_{12}^{\prime}(\tau) \\
+\left(i \frac{\Delta}{\tau_{0}^{2}}+\frac{\Delta^{2}}{\tau_{0}}+\frac{J_{B}^{2}}{2 \tau_{0}}\right) \sigma_{12}(\tau)=\frac{J_{B}^{2}}{2 \tau_{0}} \sigma_{21}(\tau),
\end{gathered}
$$

where $\sigma_{12}(\tau)=\rho_{12}(\tau) /\left|\rho_{12}(0)\right|$ is the normalized offdiagonal element of the qubit density matrix. The initial conditions are

$$
\begin{aligned}
& \sigma_{12}^{\prime \prime}(0)=\left(-\Delta^{2}-\frac{J_{B}^{2}}{2}\right) \sigma_{12}(0)+\frac{J_{B}^{2}}{2} \sigma_{21}(0), \\
& \sigma_{12}^{\prime}(0)=-i \Delta \sigma_{12}(0) \\
& \sigma_{12}(0)=e^{i \phi}
\end{aligned}
$$

where $\phi$ is the initial phase of the off-diagonal density matrix element.

\section{A. Analytic solutions}

Differential equation (27) with initial condition Eqs. (28 - 30) has explicit solutions:

$$
\begin{aligned}
\sigma_{12}(u)= & \sum_{i=1}^{3}\left[e^{\lambda_{i} u}+\frac{1}{\alpha} \lambda_{i} C_{i c}\left(e^{\lambda_{i} u}-e^{-u}\right)\right] \\
& \times\left(C_{i c} \cos \phi+i C_{i s} \sin \phi\right)+i e^{-u} \sin \phi,
\end{aligned}
$$

where $u=\tau / \tau_{0}$. For $i=1-3$, the coefficients are given by

$$
\begin{aligned}
C_{i c} & =\frac{1}{\Delta_{i}}\left(\left(1+\beta^{2}\right) \lambda_{i}^{2}\right. \\
& \left.+\left(1+\beta^{2}-2 \alpha^{2}\right) \lambda_{i}+\left(1+\beta^{2}\right) \gamma^{2}-3 \alpha^{2}\right), \\
C_{i s} & =\frac{1}{\Delta_{i}} \alpha\left(2 \lambda_{i}^{2}+3 \lambda_{i}+1+\beta^{2}\right), \\
\Delta_{i} & =\left(\lambda_{i}^{2}+2 \lambda_{i}+1+\gamma^{2}\right)\left(3 \lambda_{i}^{2}+2 \lambda_{i}+\gamma^{2}\right),
\end{aligned}
$$

and the $\lambda_{i}^{\prime} s$ are the three solutions of

$$
\lambda^{3}+\lambda^{2}+\gamma^{2} \lambda+\alpha^{2}=0,
$$

where $\alpha=\tau_{0} \Delta, \beta=\tau_{0} J_{B}$, and $\gamma^{2}=\alpha^{2}+\beta^{2}$.

We will show one special case and three asymptotes.

$\beta=0$ isolated system: Since Eq. (35) has solutions $\lambda_{1}=-1$ and $\lambda_{2,3}= \pm i \alpha$, the coefficients are determined as $C_{1 c}=C_{1 s}=0, C_{2 c}=C_{3 c}=\frac{1}{2}$ and $C_{2 s}=C_{3 s}=\frac{1}{2 i}$. Therefore, we get $\sigma_{12}(u)=e^{-i \alpha u+i \phi}$, which is simply a natural rotation of the off-diagonal element of the density matrix.

$\alpha, \beta \ll 1$ fast modulation: In this asymptotic case, the roots of Eq. (35) are $\lambda_{1} \sim-1+\beta^{2}$ and $\lambda_{2,3} \sim$ $-\frac{1}{2} \beta^{2} \pm i \alpha$. After determining coefficients $C_{i}$, we obtain

$$
\sigma_{12}(u) \sim e^{-i \alpha u-\frac{\beta^{2}}{2} u+i \phi} .
$$

Therefore, the off-diagonal element of the qubit density matrix decays exponentially at a rate of $T_{2}^{-1} \sim \frac{1}{2} J_{B}^{2} \tau_{0}$.

$\alpha, \beta \gg 1$ slow modulation: The roots are $\lambda_{1} \sim-\frac{\alpha^{2}}{\gamma^{2}}$ and $\lambda_{2,3} \sim-\frac{\beta^{2}}{2 \gamma^{2}} \pm \gamma i$, so $\Re \sigma_{12}(u) \sim \frac{1}{\gamma^{2}}\left(\beta^{2} e^{-\frac{\alpha^{2}}{\gamma^{2}} u}+\right.$ $\left.\alpha^{2} e^{-\frac{\beta^{2}}{2 \gamma^{2}} u} \cos \gamma u\right) \cos \phi+\frac{\alpha}{\gamma} e^{-\frac{\beta^{2}}{2 \gamma^{2}} u} \sin \gamma u \sin \phi \quad$ and $\Im \sigma_{12}(u) \sim e^{-\frac{\beta^{2}}{2 \gamma^{2}} u}\left(-\frac{\alpha}{\gamma} \sin \gamma u \cos \phi+\cos \gamma u \sin \phi\right)$. In particular, for $1 \ll \alpha \ll \beta$ (strong coupling limit),

$$
\begin{aligned}
\sigma_{12}(\tau) & \sim e^{-\frac{\alpha^{2}}{\beta^{2}} u} \cos \phi \\
& +i e^{-\frac{1}{2} u} \cos \beta u \sin \phi .
\end{aligned}
$$

These apparent different time dependences between the real and imaginary parts stem from the choice of coupling in the form $J_{B} \sigma_{x} X / 2$. If we choose the form $J_{B} \sigma_{y} X / 2$ instead, the time dependences of the real and imaginary parts are interchanged. In this strong coupling limit, the time evolution of $\sigma_{12}(\tau)$ explicitly depends on its initial 
phase, $\phi$, not the simply like $e^{i \phi}$. Therefore, as will be discussed later, if there are several such impurities, the total time evolution of $\sigma_{12}(\tau)$ is not the simple product of each impurity's contribution. For $1 \ll \beta \ll \alpha$ (weak coupling), we have

$$
\sigma(u) \sim e^{-\frac{\beta^{2}}{2 \alpha^{2}} u+i \phi} .
$$

In this case, we have exponential decay with $T_{2}^{-1} \sim$ $\frac{J_{B}^{2}}{2 \Delta^{2}} \frac{1}{\tau_{0}}$.

For $\alpha, 1 \gg \beta$ weak coupling or preservative regime, have $\lambda_{1} \sim-1-\frac{\beta}{\alpha^{2}+1} i$, and $\lambda_{2,3} \sim-\frac{\beta^{2}}{2\left(\alpha^{2}+1\right)} \pm$ $\left(1+\frac{\beta^{2}}{2\left(\alpha^{2}+1\right)}\right) i$, so $\sigma_{12} \sim e^{-i \alpha\left(1+\frac{\beta^{2}}{2\left(\alpha^{2}+1\right)}\right) u-\frac{\beta^{2}}{2\left(\alpha^{2}+1\right)} u+i \phi}$. Therefore, we again have exponential decay with $T_{2}^{-1} \sim$ $\frac{J_{B}^{2} \tau_{0}}{2\left(1+\Delta^{2} \tau_{0}^{2}\right)}$. This coincides with the Redfield result, which was obtained by perturbation theory and is justified in the weak coupling case, $\left|J_{B}\right| \ll 1 / \tau_{0}[23,24]$. Taking the limit $\alpha \ll 1$ further, we restore the result for $\alpha, \beta \ll 1$, fast modulation.

To summarize, the dephasing rate is given by

$$
T_{2}^{-1}=\left\{\begin{array}{cc}
J_{B}^{2} \tau_{0} / 2, & \text { for } \Delta,\left|J_{B}\right| \ll 1 / \tau_{0} \\
\Delta^{2} / J_{B}^{2} \tau_{0}, & \text { for the real part and } \\
& 1 / \tau_{0} \ll \Delta \ll\left|J_{B}\right| \\
1 / 2 \tau_{0}, & \text { for the imaginary par } \\
& \text { and } 1 / \tau_{0} \ll \Delta \ll\left|J_{B}\right| \\
J_{B}^{2} / 2 \Delta^{2} \tau_{0}, & \text { for } 1 / \tau_{0} \ll\left|J_{B}\right| \ll \Delta \\
J_{B}^{2} \tau_{0} / 2\left(1+\Delta^{2} \tau_{0}^{2}\right) & \text { For }\left|J_{B}\right| \ll \frac{1}{\tau_{0}}, \Delta .
\end{array}\right.
$$

In all regimes, $T_{2}>2 \tau_{0}$.

\section{B. Numerical results}

Here, we show the results of solving Eqs. (26) and (27) numerically. Figure 3 shows the $\tau / \tau_{0}$ dependence of $n(\tau)$ and $\left|\sigma_{12}(\tau)\right|$ when $\left|J_{B}\right| \ll \Delta, 1 / \tau_{0}$ (weak coupling) along with the asymptotic curves obtained analytically.

Figure 4 shows the $\tau / \tau_{0}$ dependence of $n(\tau)$ and $\left|\sigma_{12}(\tau)\right|$ in the case of strong coupling. It also shows the asymptotic envelope for $n(\tau)$.

Figure 5 shows the $\tau / \tau_{0}$ dependence of $\Re \sigma_{12}(\tau)$ in the case of strong coupling. It also shows the asymptotic curve. As shown in Figs. 3, 4, and 5, in the two contrasting limits, the numerical and analytical results coincide very well. It should be noted that we do not find Gaussian decay of the off-diagonal element of the density matrix for the initial regime, in contrast to the fluctuation in the tunneling coupling constant.

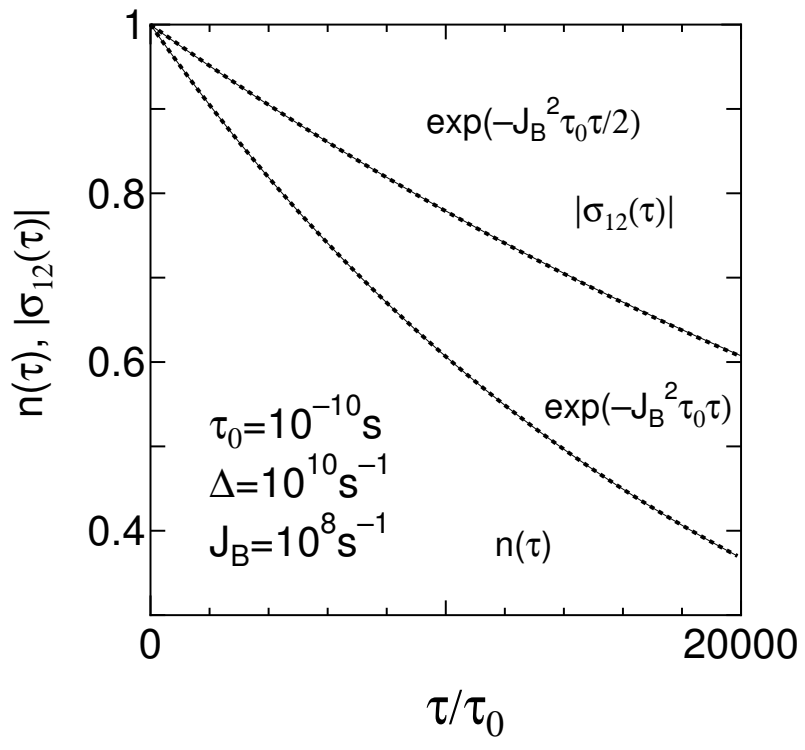

FIG. 3. The $\tau / \tau_{0}$ dependency of density matrix of $n(\tau)$ and $\left|\sigma_{12}\right|(\tau)$ (solid curve) when $J_{T}=0$ with $J_{B}=10^{8} \mathrm{~s}^{-1}$ and $\Delta=10^{10} \mathrm{~s}^{-1}$. Dotted lines are analytically obtained asymptotic curves, which is almost identical to the solid curves.

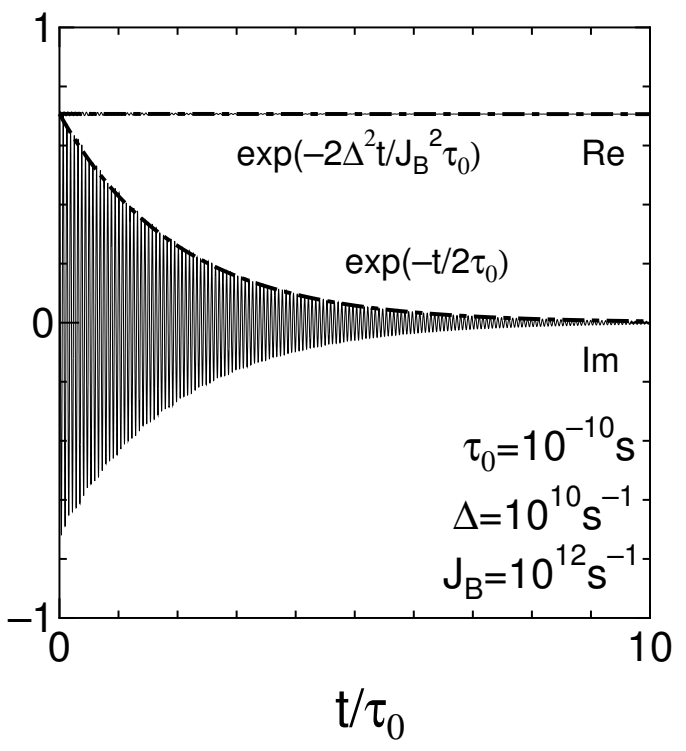

FIG. 4. The $\tau / \tau_{0}$ dependency of density matrix of $n(\tau)$ and $\left|\sigma_{12}\right|(\tau)$ (solid curve) when $J_{T}=0$ with $J_{B}=10^{12} \mathrm{~s}^{-1}$ and $\Delta=10^{10} s^{-1}$. Dotted line is analytically obtained asymptotic envelope curve. 


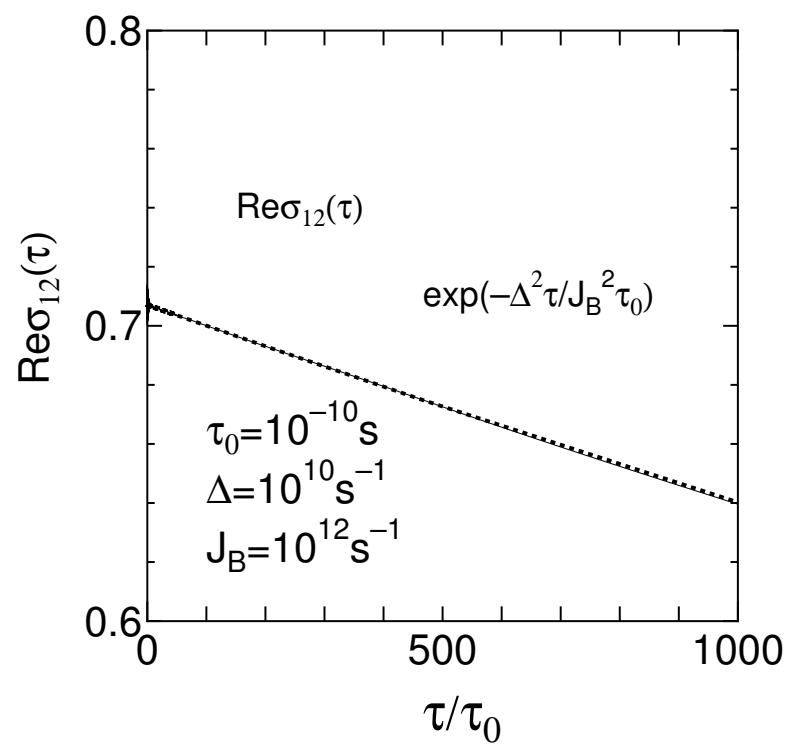

FIG. 5. The $\tau / \tau_{0}$ dependence of real part of density matrix $\sigma_{12}(\tau)$ (solid line) when $J_{T}=0$ with $J_{B}=10^{12} s^{-1}$ and $\Delta=10^{10} s^{-1}$. Dotted line is analytically obtained asymptotic curve.

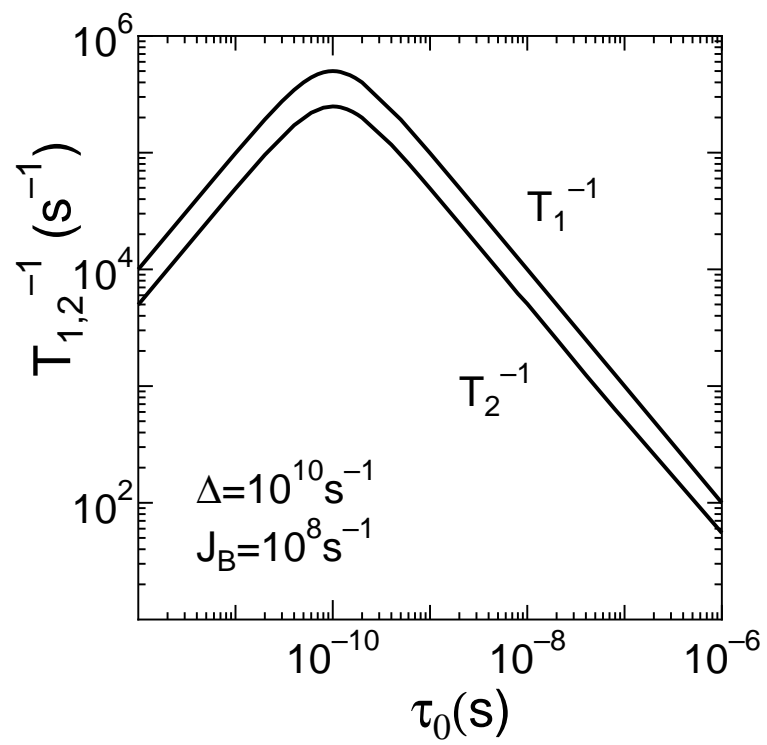

FIG. 6. The $\tau_{0}$ dependence of $T_{1}^{-1}$ and $T_{2}^{-1}$ when $J_{T}=0$ with $J_{B}=10^{8} \mathrm{~s}^{-1}$ and $\Delta=10^{10} \mathrm{~s}^{-1}$.

Next, we examine the $\tau_{0}$ dependence of the relaxation rate, particularly for $\left|J_{B}\right|<\Delta$. Figure 6 shows the $\tau_{0}$ dependences of $T_{1}^{-1}$ and $T_{2}^{-1}$ when $\Delta=10^{10} \mathrm{~s}^{-1}$ and $J_{B}=10^{8} s^{-1}$. The limits of the long and short $\tau_{0}$ fit well with the analytical asymptotic given by Eq. (39). The $\tau_{0}$ dependence of the relaxation time is fitted as

$$
T_{1}^{-1}=2 T_{2}^{-1}=\frac{J_{B}^{2} \tau_{0}}{1+\Delta^{2} \tau_{0}^{2}} .
$$

When $\tau_{0}<\Delta^{-1}$, the relaxation rates increase with $\tau_{0}$. When $\tau_{0}>\Delta^{-1}$, the rates decrease with an increasing $\tau_{0}$. The shape of the dephasing rate as a function of $\tau_{0}$ is explained as follows. When $\tau_{0}<\Delta^{-1}$, many dephasing events occur during one Rabi oscillation cycle, each event leads to collective disturbance. Because the long time constant of telegraph noise leads to large fluctuations in the variance of the rotating angle in the Bloch sphere during Rabi oscillation, the dephasing time decreases with an increasing $\tau_{0}$. When $\tau_{0}>\Delta^{-1}$, Rabi oscillation occurs over more than one cycle in time $\tau_{0}$ and, in this regime, each dephasing event is independent. Hence, the dephasing time increases with $\tau_{0}$. The maximum around $\tau_{0} \sim \Delta^{-1}$ is a kind of resonance.

\section{DISCUSSION}

We considered the effect of electrostatic disturbance due to background charge fluctuations (BCFs). To summarize our findings, for pure dephasing $\left(J_{T} \neq 0, J_{B}=0\right)$, $2 \tau_{0}<T_{2}$ and $T_{1}=\infty$. For dephasing with relaxation of the population $\left(J_{T}=0, J_{B} \neq 0\right), 2 \tau_{0}<T_{2}=2 T_{1}$.

Next, we discuss the relationship between the experimentally observed $T_{2}$ and our results. In the present study, the dephasing time with a single background charge was found to be longer than the time constant of the telegraph noise for both tunneling and bias fluctuations. The observed time constant of a dominant random telegraph noise is about $30 \mu s$ or longer [10,12], so a rather long dephasing time is expected. However, in another experiment, the dephasing time was about $1 \mathrm{~ns}$ [25]. Therefore, a single telegraph noise source may not be enough to explain the experimental results; we should thus consider the effect of many impurity sites or other additional effects.

We consider the effect of many impurities for the case in which there is no correlation between background charges. Flucuation in the tunnel coupling arises from the modulation of the wave function in the coupled dots. The gradient of the electrostatic potential around the tunneling barrier, which comes from an electron or hole located at an impurity site, leads to a change in the tunnel coupling as well as a change in the asymmetric bias [26]. For a charge state that couples with a coupleddot system symmetrically, the pure dephasing event is critical. For dephasing accompanied with relaxation of the population, the unitary operators of each impurity, which lead to dephasing of the qubit, are not commuting. However, by neglecting the higher order $J_{B} \tau_{0}$ 's in the dephasing rate, we can take the ensemble sum of the effect of each charge state. In such a weak coupling case, $\left(J_{B} \tau_{0} \ll 1\right)$, we can use Eq. (40), and the simple summation of the dephasing rate is expressed by [20]

$$
\begin{aligned}
T_{2 \Sigma}^{-1} & =\int d J_{B} P_{\Sigma}\left(J_{B}\right) \int d W P(W) T_{2}^{-1}\left(J_{B}, W\right) \\
& =\frac{k_{B} T}{W_{0}} \frac{\pi<J_{B}^{2}>_{\Sigma}}{4 \Delta}
\end{aligned}
$$


where $W_{0}$ is the distribution width of the thermal activation energy of the charge states, $P_{\Sigma}\left(J_{B}\right)$ is the distribution function of $J_{B}$, which depends on the relative position between the qubit and impurity site, and $<J_{B}^{2}>_{\Sigma}\left(=\int d J_{B} P_{\Sigma}\left(J_{B}\right) J_{B}^{2}\right)$ is the sum over the random impurities. In the second equation, we assume uniform distribution of the activation energies of the background charges, $P(W)=1 / W_{0}$; for typical cases, $W_{0} / k_{B} T$ is approximately 23 [20]. Use of the perturbation method showed that the dephasing rate of a Josephson charge qubit in terms of $J_{B} \tau_{0}$ is proportional to the inverse of $E_{J}$ in the limit of $E_{C}=0$ [14,15], where $E_{J}$ and $E_{C}$ are the Josephson coupling constant and charging energy, respectively. This is similar to the estimate of $T_{2 \Sigma}^{-1}$ in Eq. (41), where $E_{J} / \hbar$ corresponds to $\Delta$. With a larger $\Delta$ and lower temperature, the dephasing rate is lower.

We next estimate the magnitude of the fluctuations, $J_{B}$. The asymmetric bias fluctuation comes from asymmetric coupling between the two dots and the background charge, which is in the form of a dipole interaction, $J_{B} \propto e^{2} d \cos \theta / r^{2}$ for $d \ll r$, where $d$ is the distance between the two dots, $r$ is the distance between the coupled-dot system and the background charge, and $\theta$ is the angle between them [27]. Therefore, for a smaller qubit or a charge located far from the qubit, the effect of bias fluctuation should be less important. The dephasing rate is proportional to $\left\langle J_{B}^{2}\right\rangle_{\Sigma}$, which is estimated as

$$
\begin{aligned}
\sum_{i}\left(\frac{e^{2} d}{4 \pi \epsilon_{r} \epsilon_{0} \hbar r_{i}^{2}} \cos \theta_{i}\right)^{2} \sim & \left(\frac{e^{2} d}{4 \pi \epsilon_{r} \epsilon_{0} \hbar}\right)^{2} N_{i} \int_{r_{m}(d)}^{\infty} r^{2} d r \\
& \times \int_{0}^{\pi} \sin \theta \frac{\cos ^{2} \theta}{r^{4}} d \theta 2 \pi \\
& =\left(\frac{e^{2} d}{4 \epsilon_{r} \epsilon_{0} \hbar}\right)^{2} \frac{4 \pi}{3} \frac{N_{i}}{r_{m}(d)}
\end{aligned}
$$

for the impurity sites where the dipole approximation is appropriate, where $r_{m}(d)$ is the radius beyond which the dipole approximation is valid, which depends on $d, N_{i}$ is the density of impurity sites, and $\epsilon_{r}$ is the relative dielectric constant. Therefore, the total dephasing time is well defined. The quality factor of a quantum logic gate is defined by the ratio of $\Delta$ to $T_{2 \Sigma}^{-1}$ :

$$
Q=\frac{\Delta}{T_{2 \Sigma}^{-1}}=\frac{W_{0}}{k_{B} T} \frac{4 \Delta^{2}}{\pi<J_{B}^{2}>_{\Sigma}},
$$

which represents how many gate operations can be done before the quantum coherence vanishes. From Eq. (43), we conclude that a large $\frac{\Delta^{2}}{\left\langle J_{B}^{2}\right\rangle_{\Sigma}}$ is needed for quantum computation. We estimated Q using $d=0.3 \mu m, r_{m}=1$ $\mu m, \Delta=200 \mu e V$ (characteristic parameter values for an experiment in which the quantum mechanical coupling of the dots was observed in the frequency domain [5] ), and $\epsilon_{r}=12.5$ (for GaAs). To enable quantum error correction, the lower bound of the necessary gate quality factor was roughly estimated as $Q>10^{6}$ [28]. Thus, density of charge states should be less than $5 \times 10^{6} \mathrm{~cm}^{-3}$ for fabrication. If there is a correlation between impurities (a screening effect), dephasing will be suppressed in general [29]. It should be noted that there might be strong dephasing from the nearby impurities for which dipole approximation is not adequate, even if these are only a few impurities, (a few in this case). The non-commutativity between the qubit Hamiltonian and environment Hamiltonian and the qubit backaction make it difficult to evaluate the dephasing rate for strongly coupled background charge fluctuations in the asymmetric bias case [14].

Finally, we discuss the Josephson charge qubit system [1]. Under an appropriate condition (single-electron charging energy $E_{C}$ much larger than Josephson coupling energy $E_{J}$ and temperature $k_{B} T \ll E_{J}$ ) only two charge states in the Cooper pair box (CPB) are important, and the Hamiltonian is given by

$$
H=\frac{E_{J}}{2} \sigma_{x}+\frac{\delta E_{C}}{2} \sigma_{z}+\frac{\hbar \sum_{i} J_{C i} X_{i}}{2} \sigma_{z},
$$

where $\delta E_{C}=4 E_{C}\left(Q_{t} / e-1\right)$ is the energy difference between the two charge states, and $Q_{t}$ is the total gateinduced charge in the box. The two-charge-state basis is expressed using Pauli matrices, and $\hbar J_{C}$ is the coupling strength between the qubit and the background charge, which induces fluctuation in the charging energy. The $E_{J} / \hbar$ corresponds to the asymmetric bias $\epsilon$, and $E_{C} / \hbar$ corresponds to $\Delta$. Here, $E_{C} \simeq 122 \mu \mathrm{eV}$, and $E_{J} \simeq 34$ $\mu \mathrm{eV}[16]$; if we can neglect $E_{J}$, the pure dephasing event is critical. In pure dephasing, the effect of a large number of impurities is obtained by simply summing the dephasing rates, because $H_{q b}$ and interaction Hamiltonian commute. When the background charge and CPB interact, the charging energy in the CPB fluctuates. The spectrum of the fluctuation is given by

$$
\begin{aligned}
S_{\Delta E}(\omega) & =\int d t e^{i \omega t} \sum_{i j} \hbar^{2}<J_{C i} X_{i}(t) J_{C j} X_{j}(0)> \\
& =\sum_{i} \frac{\hbar^{2} J_{C i}^{2} \tau_{0 i}}{1+\omega^{2} \tau_{0 i}^{2}} \\
& =\int d J_{C} P\left(J_{C}\right) \int d \tau_{0} P\left(\tau_{0}\right) \frac{\hbar^{2} J_{C}^{2} \tau_{0}}{1+\omega^{2} \tau_{0}^{2}} \\
& \simeq \frac{k_{B} T}{W_{0}} \frac{\pi \hbar^{2}<J_{C}^{2}>}{2 \omega},
\end{aligned}
$$

where $\left\langle J_{C}^{2}\right\rangle=\int d J_{C} P\left(J_{C}\right) J_{C}^{2}$, and we take an ensemble average over the activation energy, as was done in the coupled-dot system. The spectrum of the charging energy fluctuation was experimentally found to be $S_{\Delta E}(\omega)=\left(\frac{4 E_{C}}{e}\right)^{2} \frac{\alpha}{\omega}$, where $\alpha=\left(1.3 \times 10^{-3} e\right)^{2}[16]$. From this estimation, $<J_{C}^{2}>\simeq 4.6 \times 10^{23} s^{-2}$ for $20 \mathrm{mK}$. For an initial regime, the envelope of Rabi oscillation shows Gaussian decay, namely, the off-diagonal element of the 
density matrix is given by $\rho_{12}(t) \simeq \rho_{12}(0) \exp \left(-<J_{C}^{2}>\right.$ $\left.t^{2} / 2\right) e^{-i E_{J} t / \hbar}$. The rate of Gaussian decay is given by $\sqrt{\left\langle J_{C}^{2}\right\rangle / 2}$. For the above value of $\left\langle J_{C}^{2}\right\rangle$, the time constant of the Gaussian decay is given by $3.6 \times 10^{2} \mathrm{ps}$, which is consistent with the experimental finding of 150 $p s$, [16]. Note that in the Gaussian regime, the time constant does not depend on the temperature. Numerical calculation [14] also suggests this type of Gaussian decay for the pure dephasing case.

At the charge degeneracy point, namely $\delta E_{C}=0$, dephasing with relaxation of the population occurs. In this case, the dephasing rate is estimated using the same value of $\left\langle J_{C}^{2}>\right.$ :

$$
T_{2}^{-1}=\frac{\pi}{4} \frac{k_{B} T}{W_{0}} \frac{<J_{C}^{2}>}{E_{J}}
$$

so $T_{2} \simeq 0.28 \mu \mathrm{s}$ for $T=20 \mathrm{mK}$. In a recent experiment, a longer coherence time of $0.50 \mu \mathrm{s}$ was found when the saddle point of the ground state energy was used as a function of $Q_{t}$ and the flux [30].

\section{SUMMARY}

We examined the effect of the fluctuation of a single charge in an impurity site on a qubit. Using the method of stochastic differential equations, we calculated the time evolution of the ensemble averaged density matrix of the qubit and obtained analytical results for various conditions. The dephasing time, $T_{2}$, was always longer than the time constant of the random telegraph noise for both tunneling and bias fluctuations. For bias fluctuation, $T_{2}$ was twice the relaxation time of the population in the weak coupling case. To suppress the bias fluctuation, the coupled dots should be positioned closer together or the tunnel coupling should be made stronger. We also investigated the case in which many impurity sites are distributed and examined the gate quality factor. For pure dephasing, which corresponds to a Josephson charge qubit experiment, the Gaussian decay of the off-diagonal element of the density matrix dominated. The present results can be applied to other quantum two-level systems in which there is telegraph-type fluctuations.

Acknowledgements We thank J. S. Tsai, T. Tanamoto, N. Imoto, M. Koashi, H. Nakano, T. Fujisawa, Y. Hirayama, T. Hayashi, C. Uchiyama and K. Harmans for their stimulating discussions. This work was partly supported by CREST-JST, Interacting Carrier Electronics Project.
[1] Y. Nakamura, Yu. A. Pashkin and J. S. Tsai: Nature 398 786 (1999).

[2] D. Loss and D. P. DiVincenzo: Phys. Rev. A 57120 (1998).

[3] T. Tanamoto: Phys. Rev. A 6122305 (2000).

[4] A. Miranowicz, S. K. Özdemir, M. Koashi, N. Imoto and Y. Hirayama: Phys. Rev. A 65062321 (2002).

[5] T. H. Oosterkamp, T. Fujisawa, W. G. van der Wiel, K. Ishibashi, R. V. Hijman, S. Tarucha and L. P. Kouwenhoven: Nature 395873 (1998).

[6] T. Fujisawa, T. H. Oosterkamp, W. G. van der Wiel, B. W. Broer, R. Aguado, S. Tarucha and L. P. Kouwenhoven: Science 282932 (1998).

[7] A. Shnirman, G. Schön and Z. Hermon: Phys. Rev. Lett. 792371 (1997).

[8] P. L. Lafarge, P. Joyez, H. Pothier, A. Cleland, T. Holst, D. Esteve, C. Urbina and M. H. Devoret: C. R. Acad. Sci. Paris, 314883 (1992).

[9] G. Zimmerli, T. M. Eiles, R. L. Kautz and J. M. Martinis: Appl. Phys. Lett. 6113 (1992).

[10] C. Kurdak, C. J. Chen, D. C. Tsui, S. Parihar, S. Lyon and G. W. Weimann: Phys. Rev. B 569813 (1997).

[11] A. B. Zorin, F.-J. Ahlers, J. Niemeyer, T. Weimann, H. Worf, V. A. Krupenin and S.V. Lotkhov: Phys. Rev. B 5313682 (1996)

[12] T. Fujisawa and Y. Hirayama: Appl. Phys. Lett 77543 (2000).

[13] P. Dutta and P. H. Horn: Rev. Mod. Phys. 53497 (1981).

[14] E. Paladino, L. Faoro, G. Falci and R. Fazio: Phys. Rev. Lett. 88228304 (2002).

[15] A. Shnirman, Y. Makhlin and G. Schön: condmat/0202518.

[16] Y. Nakamura, Yu A. Pashkin, T. Yamamoto and J. S. Tsai: Phys. Rev. Lett. 88047901 (2002)

[17] In the present study, dissipation means the relaxation of the population of bonding-antibonding states. On large time scales, the qubit state becomes mixed with equal populations of bonding and anti-bonding states, this relaxation is not an energy relaxation.

[18] Y. M. Galperin and K. A. Chao: Phys. Rev. B 5212126 (1995).

[19] R. Bauernschmitt and Yuli V. Nazarov: Phys. Rev. B 47 9997 (1992).

[20] Y. Imry: Introduction to Mesoscopic Physics, Oxford University Press.

[21] A. I. Burshtein: Soviet Phys. JETP 22939 (1965).

[22] A. I. Burshtein: Soviet Phys. JETP 21567 (1965).

[23] A. J. Leggett, S. Chakravarty, A. T. Dorsey, M. P. A. Fisher, A. Garg and W. Zwerger: Rev. Mod. Phys. 591 (1987)

[24] C. L. Slichter: Principals of Magnetic Resonance 3th ed, Springer-Verlag.

[25] T. Fujisawa, W. G. van der Wiel and L. P. Kouwenhoven: Physica E 7413 (2000).

[26] G. Burkard, D. Loss and D. P. DiVincenzo: Phys. Rev. B 592070 (1999).

[27] In the present study, we assume the interaction between the coupled-dot system and charge state to be dipolemonopole coupling. When the electron reservoir screens the charge state effectively, the charge state and the reservoir act on the system as a dipole. The interaction type 
is dipole-dipole, and the interaction strength decreases more rapidly as the distance between the charge state and system increases. A longer dephasing time is thus expected compared with that of a dipole-monopole interaction.
[28] J. Preskill: Proc. R. Soc. London A 454385 (1998).

[29] T. Itakura and Y. Tokura: unpublished.

[30] D. Vion, A. Aassime, A. Cottet, P. Joyez, H. Pothier, C. Urbina, D. Esteve and M. H. Devoret: Science 296886 (2002). 